\title{
Medicine, Behavior and Social Values: Exploring the Practical Range of Sociopharmacology
}

\author{
John H. Morgan* \\ Senior Fellow in Behavioral Science, Foundation House/Oxford, UK
}

*Corresponding author: John H. Morgan, Senior Fellow in Behavioral Science, Graduate Theological Foundation (US), Dodge House 415 Lincoln Way East, Mishawaka, Indiana, Tel: +465 441-800-423-5983; E-mail: faculty@gtfeducation.org

Citation: Morgan JH (2016) Medicine, Behavior and Social Values: Exploring the Practical Range of Sociopharmacology, J Pharma Pharma Sci 2016: G 110. DOI:10.29011/2574-7711/100010

Revived Date: 11 November 2016; Accepted Date: 15 November 2016; Published Date: 22 November 2016

The field of Sociopharmacology is defined as the study of the behavioral relationship between pharmaceuticals, i.e., compounds manufactured for use as a medicinal drug, and social behavior with special reference to social values affecting medicine consumption and usage [1]. Illustrative of Sociopharmacology is the study of the relationship of over-the-counter pain medication and the social value of pain tolerance within a society or the relationship of over-the-counter diet medication and the social value of thinness dictated by society and quite recently the relationship of over-thecounter teeth treatment and the value placed on their whiteness by a society. A whole field of study in Sociopharmacology is the relationship between athletic medications and social values of a society immersed in sports and the sporting industry of special interest is performance expectations of the individual as those expectations are mediated through the social values of a particular society [2].

Psychopharmacology is, of course, the study of the use of psychiatric drugs in the treatment of psychological illness [3]. Sociopharmacology, on the other hand, focuses its attention upon the behavioral relationship between pharmaceuticals (both over-thecounter drugs and those prescribed by a medical practitioner) and social values [4]. That is to say, whereas psychopharmacology addresses the interplay between medication and behavior, Sociopharmacology addresses the interplay between medication and social values [5].

I first used the term "Sociopharmacology" in 1981 in a medical sociology course at Saint Mary's College at Notre Dame, Indiana. The following year, I published a research bibliography titled Sociopharmacology. However, one of the earliest uses of the term "Sociopharmacology" I have been able to find is an article by and again in $[6,7]$. However, a substantially more developed essay by L. von Ferber titled "What is Social Pharmacology: What Are Its Goals?" appeared in a German publication in [8] where he writes: "Sociopharmacology is based on interdisciplinary research involving pharmacology, epidemiology, social medicine and sociology. It aims at finding out the social determinants of drug intake. How this goal is approached stepwise is shown in the case of the benzodiazepine group of active substances." I am convinced that Professor von Ferber's definition is useful in so far as it goes but it is my experience in the field that this definition is too restrictive and impedes a wider range of research interests covered in my definition.

As both a clinical psychotherapist and social psychologist, I make a justifiable distinction between the study of medication access and social values (called Sociopharmacology) and the study of psychiatric drugs in the treatment of mental illness (called psychopharmacology). Correlations between medication and social values worthy of empirically-based data studies include such areas as diet supplements and pain medications and the social values implied in their use by consumers $[9,10]$ has demonstrated the effective use of Sociopharmacology in the study of "disparity" within society relative to the role and function of ethnicity and race, socioeconomic stratification, and physical and behavioral characteristics of tobacco users. Though the complex matrices of variables related to and affecting a comprehensive analysis of this consumer-based addictive commodity is substantial, he suggests that traditional psychopharmacology has not addressed itself to the subject with any significant degree of rigor and insightfulness. His call for a more sustained transdisciplinary and multidisciplinary address to the relationship between social values and addictive substance access is right in keeping with the rise in the utility of sociopharmacological methodology.

Clearly, both social and cultural contexts as well as social values come into play in the user/provider matrix of legal addictive consumption and here is where Sociopharmacology, as a compliment and enhancement of psychopharmacology, demonstrates its analytical utility. Though clearly out of touch with other major 
sociopharmacological research in other fields outside tobacco use, indicated in his amazingly naïve reference to Sociopharmacology as "a novel paradigm," nevertheless, his definition of Sociopharmacology is practically functional however limited in scope. Though extremely limited in scope and thus crippled in its more expansive possibilities, he defines it as "a platform for investigating how contextual factors amplify psychopharmacological determinants of smoking to disproportionately enhance vulnerability to smoking in populations subject to TRHD (Tobacco-Related Health Disparities)."

What is both gratifying and disappointing about Leventhal's use of the term and methodology of Sociopharmacology is, first, he has elevated its recognizable contribution as an analytical tool and methodology, while, second, limiting its utility and expansive applicability to a much broader matrix of pharmaceuticals and social values interaction. Nevertheless and more on this point later, he does four very important things in this key article within the confines of this limited use of Sociopharmacology. Beyond restating the relevance of this overarching methodology of medical access and social values and providing illustrative materials within tobacco-using populations of this approach's utility, he shows how this approach accentuates the applicability of Sociopharmacology to the study of the public impact additive drugs have while interfacing with social values, and, finally, he points to the praxis possibilities of Sociopharmacology in public policy formation and intervention in consumer behavior relative to addictive stimulants generally [10].

As discussed by [11], psychopharmacology addresses behavioral matrices related to pharmaceutical interaction and intervention whereas we are suggesting here that Sociopharmacology rather focuses its analytical prowess upon the relationship between society (and its social values) and access and utilization of pharmaceuticals. Clearly, since behavior and social values are integrally related, it only stands to reason that an analytical methodology which addresses either behavior or social values must necessarily be related. Friedman, however, is eager to point out that since psychopharmacology defines the "user as the problem" with reference to addictive drug use, for example, this approach, insightful and effective as it may be, nevertheless overlooks the social matrices - economics, social class, education, etc. - which constitutes the context of users vulnerable to drug misuse.

Though still delimiting the application of sociopharmacological research to issues related to drug use (rather than to broader societal issues and values as I have been contending should be the case), Friedman nevertheless does demonstrate how he has chosen to apply the sociopharmacological analysis of drug use to a broader context that is provided by psychopharmacology. In this major article in the field of Sociopharmacology, [11] provides six levels of application beginning with data on drug-related harm to social complexities and contradictions within the society matrix of use and abuse, suggesting that contrary to the inclination of psychopharmacology to lay blame on the user the accusation of blame and fault should rest with the social order itself. Furthermore, while acknowledging that individual, family and social harm results from drug misuse and abuse, he contends that the legal punishment of users as well as dealers are lamentably founded on the bogus blame on the user rather than the social contextualization of user-culture thereby providing an easy out, a "scapegoat," for both politicians and legislation.

A more realistic and socially responsible approach, Friedman argues, should be a redirection of research towards the social, economic and public health policies which exacerbate and, alas, too often contribute to the increase in drug use, misuse, and abuse. Providing alternative outlets for social conditions contributory to drug use culture should very well constitute the primary focus in public policy, seeking avenues of social change rather than blaming and punishing the drug user, misuse, and abuser. Though limiting his focus to illegal drug use culture, clearly his paper demonstrates the potential for a substantial address to the wider field of social values as relates to legal, over-the-counter medications and pharmaceuticals.

It is lamentable that the broadly conceived application of sociopharmacological research envisioned by researchers at the University of California at Los Angeles [12], over 30 years ago has somewhat fallen by the wayside in deference to the noble but limiting application of this analytical perspective to a rather narrowly circumscribed field of studying in the area of addictive drugs and tobacco. They proposed (and demonstrated) the identification, organization, and evaluation of behavioral data subject to sociopharmacological analysis, providing further a paradigm for demonstration of the effectiveness of this analytical tool and methodology. While providing a summary of the UCLA research strategies and data analysis, they emphasized the initial impetus for this approach when they became convinced of the interconnectedness of pharmaceuticals such as psychotropic drugs and the behavioral matrix of individuals within a socially identifiable context. The disconnect between the behavioral situation of the individual consumer and the individual's social environment-economic, demographic, educational, etc. - became increasingly clear.

Illustrative of the limited but highly effective circumscription of the scope of Sociopharmacology is the University of Southern California Health, Emotion and Addiction Laboratory (USCHEALabortory) [13]. Which focuses its attention upon addictive drugs, particularly tobacco, alcohol, and psychotropics. Their interest in psychoactive drugs which are mood-altering is illustrative of the social problems generated in a pharmaceutically-aware society desirous of addressing behavioral issues over which the individual may have jurisdiction with help from over-the-counter 
medications as well as legal (and illegal) addictive agents. The USC laboratory (USCHEALab) has chosen to focus its research attention upon the societal context within which drug use, misuse, and abuse occurs with special interest in addictive agents. Their awareness of and attention given to the socio-cultural, economic factors and behavioral matrices leading to this public issue is to be applauded. Their research agenda, called "Sociopharmacology," has unfortunately chosen to circumscribe and limit this otherwise broadly conceived methodological approach to the study of social value issues and pharmaceuticals to what they have chosen to describe as studies in "why, how, and for whom drugs are addictive."

Admirable as this agenda is, the USC laboratory proceeded early on in shackling Sociopharmacology with a well-thought-out definition designed to serve their own institutional agenda of addiction studies rather than allowing for a broader application of Sociopharmacology to the field of social values and pharmaceutical access, use, and abuse. In their institutional mission statement, the USCHEALabortory suggests that "Sociopharmacology applies field-based correlation research, lab-based experimental psychopharmacology research, and ecological momentary assessment to examine individual differences and contextual moderators of the mood-altering effects of drugs."There is no quarrel here with this agenda. The quarrel is with the implication that Sociopharmacology is limited to this agenda. The study of addictive agents is admirable and needed. However, what about the study of the relationship between pharmaceuticals and athletics, diet, pain management, sexual performance, for examples, illustrative of social values operative within the zeitgeist of society? Investigating cultural differences in the use of pharmaceuticals, particularly addictive agents, within the context of such variables as age, gender, ethnicity, age, socioeconomic status and mental health should go far beyond, while including, the study of addictive behavior.

Clearly one of the finest articles on the expansive application of Sociopharmacology to appear in recent days is this splendid article by [14] at the School of Medicine and Health Sciences at the University of Alcala de Henares in Madrid, Spain. What is so refreshing about Professor Alloza's paper is his ready willingness, even eagerness, to point out the broad application of this method of studying the relationship between society (and social values) and the pharmaceutical industry and the medicines it produces. He readily affirms that "social pharmacology is a field of health science which generates knowledge about marketed drugs in actual use..." This is far above and beyond the limited scope of what many in the field we have been discussing here would propose or imagine. Furthermore, Alloza suggests that this methodological approach focuses upon the evaluative process involved in assessing what he calls the "social consequences" of people's access to legally marketed drugs including components of their utilization in the lives of consumers.

Recognizing and referring to Sociopharmacology (also referenced as "Social Pharmacology" as a discipline within itself [3], is quick to emphasize that the intent of this methodological analysis of the relationship between society and marketed drugs (pharmaceuticals) is to increase our knowledge and understanding of the uses (and abuses) of over-the-counter medications after they have been developed and marketed, pointing out that the information needed about new medications following regulatory approval compliance is equally important, if not more so, than prior to its release on the market [15]. He points out that the "post-marketing period" necessarily proves a much more extensive occasion for studying its impact upon society during its post-release "social life cycle" because the post-approval context, being extremely complex, includes the fact that the regulatory development controls are absent as well as industrial management and sponsorship. Furthermore, he mentions that the health delivery agencies and regulatory bodies are somewhat unpredictable in monitoring and follow-up on usage practices by the consumer.

Herein is found a true and viable agenda for Sociopharmacology, namely, to track and monitor the impact new medications have upon public health broadly defined since this discipline is particularly eager to function as a uniquely situated mechanism for gathering usage data on market-based drugs. Identifying the broad range of potential interactions among the various components of manufacturing/marketing/consumption, empirically-based observations and conclusions can be made with precision regarding marketed drugs and their consumers including "risk-benefit factors, need for alert responses, proposed actions for decision-making, importance of negative drug effects, and promotion of proper and efficient use of drugs [14]. Alloza's essay has, more so than any other recent studies in the field, demonstrated both the viability and effectiveness of Sociopharmacology as a research method and discipline addressing a broad range of topics in the societyand-pharmaceutical matrix of public health and consumer-related behavioral issues.

Illustrative of the narrowly-applied perspective on the methodological utility of Sociopharmacology is the study recently released [16].Though not suggesting that this is the only application of Sociopharmacology as a method of analysis, it is somewhat lamentable that Hitsman does not at least acknowledge the broader range of application, rather focusing solely upon this method of analysis as relates to tobacco addition. His cross-cultural study of tobacco use decline in developing societies as compared to the U.S. where tobacco use is at a historically low level reflected in a $3.8 \%$ decline from 2009 to 2014 , suggests that preliminary data implies that the U.S. national rate of usage may have dropped below $15 \%$ last year. The conclusion or concern drawn from this narrow study, he suggests, is that tobacco use may, alas, we portrayed as 
no longer a major national health issue owing to this anticipated drop. Be that as it may, the broad issues related to society and drug use (legal addictives included) could and should have been seen as a broader application of sociopharmacological analysis.

Jose-Luis Alloza of the Biomedical Science department of the School of Medicine and Health Sciences at The University of Alcala de Henares in Madrid, Spain, was joined by Rituparna Maiti of the Department of Pharmacology at the All India Institute of Medical Sciences in Bhubaneswar, Odisha, India, to demonstrate the broad application of this newly emerging methodological approach to the study of the relationship between social issues and pharmaceuticals. One of their early research interests was the rising expectations and demands of patients taking charge of their own decision-making with respect to drug consumption [17]. Realizing that a study of marketed drug products incorporate factors affecting society, individuals, pharmaceutical industry, drug development and administrative monitoring of uses and misuses of over-the-counter medication, Sociopharmacology constitutes a merging of traditional pharmacology and sociology was inevitable. Not being restricted to or obliged to defer to clinical pharmacology owing to its exemption from Phase IV studies, Sociopharmacology addresses a broader range of issues and its applicable parameters including very importantly knowledge about marketed drugs, their distribution and consumption. It is clearly a scientific method and agenda that addresses issues involving the post-marketing of medications, what is now thought of as the "life cycle" of any marketed pharmaceutical product and, quite distinctly, addresses issues and behavioral matrices which fall outside the parameters of the drug pre-market development process.

Herein lies the broad-based range of application of Sociopharmacology and, while not even attempting to pre-empt psychopharmacology or clinical pharmacology, Sociopharmacology clearly has a major role to play in the study of the relationship of people to drugs, social behavior to pharmaceuticals [18]. Point out that "Social pharmacology or Sociopharmacology, a relatively new field in clinical pharmacology, depicts the relationships between society and drugs. In its development, the name Sociopharmacology was coined in 1960s when investigators realized that it was necessary to assess the effects of drug addiction on the mood and behavior of individuals in social settings (primarily psychotropic and drug abuse agents)." However, whereas initially this methodology was used narrowly in the study of addictive behavior, the new trend in research is to more broadly conceive and define the utility of this approach to the study of society and medication with a specific application to the expansive use of over-the-counter medications in the post-marketing period of the drug's life cycle. Alloza and Maiti conclude by saying that "this discipline has expanded its horizon and enriched the specialty by incorporating the contributions from physicians [19], pharmacists, nurses, biologists, drug epidemiolo- gists, health economists, lawyers, regulators, insurance specialists, and communications specialists" [20].

\section{References}

1. Venulet J (1985) Towards Social Pharmacology. In: Alloza JL, editor. Clinical and Social Pharmacology. Post Marketing Period. Aulander, Germany: Editio Cantor 129- 139.

2. Alloza JL (2000) "Social Pharmacology, a New Discipline" (Tribune), DiarioMédico (The Medical Journal, Madrid, Spain) 8.

3. Alloza JL (2006) Social pharmacology: The discipline that studies the marketed drug and its environment. Doctor (El Medico) 970: 9-14.

4. Alloza JL (1984) Family medicine, epidemiological drug research and social pharmacology. Med Clin (Barc) 82: 124-129.

5. Alloza JL (2003) Social pharmacology, the tip of the iceberg in public health. Methods Find ExpClinPharmacol, 25 Suppl A: 175.

6. Venulet, Jan (1976) “La pharmacologiesocialeexiste-t-elle?”Méd.Hyg 34: $435-436$

7. Venulet, Jan (1978)“Aspects of Social Pharmacology," Ernst Jucker, Editor, Chapter One in Progress in Drug Resaerch / Fortschritte der Arzneimittelforschung/Progresdes recherchespharmaceutiques 9: 25.

8. Ferbert, von L (1989) "What is Social Pharmacology: What Are Its Goals?" OffentlGesundheitswes 51: 585-591.

9. Montagne M (2004) Social pharmacology: Integrating pharmaceutical and social science research on drug effects. Drug Inf J 38: 315-20.

10. Leventhal, Adam M (2016) "The Sociopharmacology of Tobacco Addiction: Implications for Understanding Health Disparities." Nicotine Tobacco Research 18: 110-121.

11. Friedman, Samuel R (2002) "Sociopharmacology of Drug Use: Initial Thoughts," International Journal of Drug Policy 5: 341-347.

12. McGuire, Raleigh MT, Brammer MJ, GL (1982) "Sociopharmacology," Annual Review of Pharmacology and Toxicology 22: 643-661.

13. University of Southern California Health, Emotion \& Addiction Laboratory https://heal.usc.edu/https:/heal.usc.edu/about_our_research.php

14. Alloza, Jose-Luis (2014) "Social Pharmacology: A further Step in Public Health," The Open Social Science Journal 6: 8-15.

15. Alloza JL (2004) Social pharmacology: Conceptual remarks. Drug Inf J 38: 321-329.

16. Hitsman B (2016) A new blueprint for addressing tobacco use disparities to reduce health disparities: The Sociopharmacology Theory of Tobacco Addiction. Nicotine and Tobacco Research 18: 109.

17. Alloza JL, Maiti, Rituparna (2014) "Social Pharmacology: Expanding Horizons," Indian Journal of Pharmacology 46: 246-250

18. Lennard HL (1964) A Proposed Program of Research in Sociopharmacology. In: Leiderman PH, Shapiro D, editors. Psychobiological Approaches to Social Behaviour. California, USA: Stanford University Press 127- 37.

19. Montastruc JL (2002) Social pharmacology: A new topic in clinical pharmacology. Therapie 57: 420-426.

20. Alloza JL (1985) Epidemiological drug surveys in the ambulatory care environment. In: Alloza JL, editor. Clinical and Social Pharmacology. Postmarketing Period. Aulander, Germany: Editio Cantor 38- 56. 